\title{
Reading and lexical-decision tasks generate different patterns of individual variability as a function of condition difficulty
}

\author{
Pierluigi Zoccolotti $^{1,2}$ - Maria De Luca ${ }^{3}$. Gloria Di Filippo ${ }^{4}$ Chiara Valeria Marinelli ${ }^{5}$. \\ Donatella Spinelli ${ }^{3,6}$
}

Published online: 9 June 2017

(C) Psychonomic Society, Inc. 2017

\begin{abstract}
We reanalyzed previous experiments based on lexical-decision and reading-aloud tasks in children with dyslexia and control children and tested the prediction of the difference engine model (DEM) that mean condition reaction times (RTs) and standard deviations ( $S D \mathrm{~s})$ would be linearly related (Myerson et al., 2003). Then we evaluated the slope and the intercept with the $x$-axis of these linear functions in comparison with previously reported values (i.e., slope of about 0.30 and intercept of about $300 \mathrm{~ms}$ ). In the case of lexical decision, the parameters were close to these values; by contrast, in the case of reading aloud, a much steeper slope $(0.66)$ and a greater intercept $(482.6 \mathrm{~ms})$ were found. Therefore, interindividual variability grows at a much faster rate as a function of condition difficulty for reading than for lexical-decision tasks (or for other tasks reported in the literature). According to the DEM, the slope of the regression that relates means and $S D$ s indicates the degree of correlation among the durations of the stages of processing. We propose that the need for a close coupling between orthographic and
\end{abstract}

Pierluigi Zoccolotti

pierluigi.zoccolotti@uniroma1.it

1 Department of Psychology, Sapienza University of Rome, Rome, Italy

2 ISTC Institute for Cognitive Sciences and Technologies, CNR, Rome, Italy

3 Neuropsychology Unit, IRCCS Fondazione Santa Lucia, Rome, Italy

4 Unicusano University, Rome, Italy

5 Laboratory of Applied Psychology and Intervention "DREAM", Department of History Society and Human Studies, University of Salento, Lecce, Italy

6 Department of Human Movement Sciences and Health, University of Rome "Foro Italico,", Rome, Italy phonological processing in reading is what drives the particularly strong relationship between performance and interindividual variability that we observed in reading tasks.

Keywords Dyslexia $\cdot$ Vocal reaction time $\cdot$ Difference engine model $\cdot$ Reading

Evidence deriving from reaction time (RT) experiments has indicated that with increasing group mean RTs there is a corresponding increase in interindividual variability, as indexed by standard deviations $(S D \mathrm{~s})$. According to Wagenmakers and Brown (2007), this relationship is so general that it marks a law characterizing the decision times under timed conditions.

The covariation between mean RTs and $S D$ s is also a key feature of models that try to account for variation of responding times, such as the rate-and-amount model (RAM; Faust, Balota, Spieler, \& Ferraro, 1999) or the difference engine model (DEM; Myerson, Hale, Zheng, Jenkins, \& Widaman, 2003). According to these models, differences in speed of processing among individuals are expressed better in terms of global, molar variations across several tasks than in terms of a particular experimental condition. Because we were interested in describing the global factor here, we performed our analyses with the DEM, which focuses especially on this issue.

The role of global components of group differences in speed is particularly clear when the effect of aging or traumatic brain injury is examined (e.g., Puopolo, Martelli, \& Zoccolotti, 2013). For example, older adults are slower than younger adults on most tasks (e.g., Cerella, Poon, \& Williams, 1980; Verhaeghen, 2013), and this slowing can be parsimoniously expressed as a single multiplicative factor (i.e., the slope of the linear regression between the condition means of the two groups. Thus, group differences in RTs grow steadily as a function of condition difficulty over and above the specific 
characteristics of a given condition (referred to as the overadditivity effect). However, two groups may not differ from each other by the same amount across all cognitive tasks. Thus, older individuals are much more delayed in visuospatial than in lexical-linguistic tasks (Hale \& Myerson, 1996; Lawrence, Myerson, \& Hale, 1998; Verhaeghen, 2013). These effects are global (i.e., non-task-specific) in that a single multiplicative factor accounts for performance in all lexical tasks, and another one accounts for all visuospatial tasks. At the same time, aging does not exert an across-the-board influence with performances being equally modified across all tasks. The DEM refers to this pattern of findings in terms of "domains" - that is, clusters of tasks (e.g., lexical or visuospatial) within which performance can be predicted by a single (multiplicative) factor. Note that the model does not assume that any specific class of tasks constitutes a domain a priori; rather, it is assumed that domains may be defined on the basis of their functional properties.

Global factors and domain effects have been described also in the case of developmental dyslexia, which is the focus of the present article. Dyslexic children are slower than typically developing children, and a single multiplicative factor expresses this group difference well across many orthographic tasks (e.g., Zoccolotti, De Luca, Judica, \& Spinelli, 2008). In particular, dyslexics are consistently impaired in all conditions that require reading words and pseudowords as well as performing lexical-decision tasks (Paizi, De Luca, Zoccolotti, \& Burani, 2013). By contrast, over and above the general difficulty of tasks, impairment is considerably less in tasks that require processing single letters or bigrams (De Luca, Burani, Paizi, Spinelli, \& Zoccolotti, 2010) or absent, as in picture naming (Zoccolotti et al., 2008) or in lexical tasks with acoustically presented stimuli (Marinelli, Angelelli, Di Filippo, \& Zoccolotti, 2011). We propose that children with dyslexia are globally impaired in a domain characterized as the "processing of visually presented orthographic strings".

A key feature of the DEM is that for all experimental tasks, there is a linear relationship between means and SDs. This linear relationship is interpreted as due to the correlation among the durations of the processing steps involved in the performances. Accordingly, for some individuals, all processing steps in a task tend to have a brief duration, whereas for other individuals, they tend to take longer than average. Note that the DEM does not focus on specific characteristics of processing stages; by contrast, it considers only generic steps of processing for the purposes of mathematical treatment. Through various simulations, Myerson et al. (2003) proposed that increases in the correlation between steps produce a corresponding increase in the size of the slope relating mean RTs and SDs. With correlations of at least 0.30 the relationship between means and $S D$ s linearizes.

Myerson et al. (2003) computed regressions from a number of previous studies and noticed that the data from a variety of linguistic and visuospatial conditions produced slopes relating mean RTs and SDs that were close to 0.30 , as well as intercepts on the $x$-axis of about $300 \mathrm{~ms}$. Notably, these parameters held across different domains as well as across groups showing different processing speeds. Thus, data fitting showed that the same linear relationship held for lexical and visuospatial tasks as well as for younger and older adults (see in particular Fig. 14 in Myerson et al., 2003). More recently, these authors collected new data as an a priori test of the model (Chen, Hale, \& Myerson, 2007) and obtained consistent results.

Although Myerson et al. (2003) empirically observed that a slope of about 0.30 fits data from a very large variety of tasks across different studies, their interest was to support the plausibility of a single general rule rather than checking whether a single parameter of the relationship (i.e., a slope of 0.30 ) would hold across all conceivable conditions. Thus, the values of the parameters that define the relationship between means and $S D$ s in different clusters of tasks (we are especially interested here in reading), as well as in different populations such as children and pathological conditions (in particular, developmental dyslexia), remain an open question.

Indeed, in our studies on global components in developmental dyslexia we repeatedly observed that the slope relating mean vocal RTs and SDs in reading tasks is higher than 0.30 . Furthermore, we have noted that tasks putatively mapping different domains produce different slopes in Brinley plots and different relationships between means and SDs. As we reported above, in De Luca et al.'s (2010) study, children with dyslexia were severely impaired in comparison to typically developing children across word and pseudo-word reading tasks (Brinley plot: slope $=2.09$ ) but were much less impaired on letter and bigram tasks (slope $=1.22$ ). However, the two sets of tasks also yielded different linear relationships between means and $S D$ s (word and pseudo-word tasks: slope $=0.70$; letter and bigram tasks: slope $=0.40$ ). Overall, consistent with the DEM, we observed linear relationships between means and SDs; however, the parameters of this relationship varied with the task.

In the present study, we reexamined several previous experiments in which we compared dyslexic children and sameage control children in various reading-aloud and lexicaldecision tasks - that is, two critical tasks for models of word recognition (Yap \& Balota, 2015). In general, we wished to examine whether, consistent with the DEM and previous studies of a variety of different tasks with young and older adult participants (Myerson et al., 2003), reading and lexicaldecision tasks produce a consistent linear relationship between means and $S D$ s in children with and without a reading deficiency. The evidence from previous studies on this point has been mixed, particularly with respect to developmental dyslexia (e.g., Marinelli et al., 2011; Paizi et al., 2013), and we sought to resolve this issue and to clarify the reasons for any discrepancies. We also examined whether, in keeping with our previous observations, the linear relationship between means 
and SDs in reading-aloud tasks would have a steeper slope than the 0.30 reported in adults for a variety of tasks, not including reading (Myerson et al., 2003).

\section{Method}

For lexical-decision tasks, we traced five different studies (marked with asterisks in the Reference list) that included lexical-decision conditions. These involved a total of 102 dyslexic and 226 control children attending the third to the sixth grade; they were tested in 67 experimental conditions. In all cases, a letter string was presented, and the child had to indicate whether it was a word or a pseudoword by pressing one of two keys. Table 1 reports the basic information from these studies, such as the list of conditions and the relative numbers of children.

For reading we refer to our recent reanalysis of studies focusing on vocal RTs, comparing dyslexic and typically developing children (please refer to Zoccolotti, De Luca, \& Spinelli, 2015). We considered data from 11 different studies (for a total of 69 experimental conditions), involving 331 typically developing children and 172 dyslexic children attending sixth grade. Five of these studies also included 21 conditions in which pseudowords were used (data are reported in Table 2). Hence, as a control we replicated analyses also including these pseudoword conditions. In all reading studies, the vocal RTs were measured by presenting the target in the center of a computer screen; the target was visible until the children started uttering it. The RT was measured as the interval between stimulus onset and vocal onset.

In both the lexical-decision and reading-aloud studies, the experimental conditions manipulated a number of variables, including stimulus length, word frequency (in both mixed-list and pure-list contexts), the presence/absence of graphemes with contextual rules, and morphological complexity. In the present analysis, the impact of specific experimental manipulations was not taken into consideration.

\section{Results and discussion}

Figure 1 plots the SDs of dyslexics and control children as a function of the condition means for the lexical-decision tasks. The regression line fitting the data $\left(y=0.37 x-80.2, R^{2}=.73\right)$ is in keeping with data previously reported for younger and older adults by Myerson et al. (2003). In particular, the slope approximates the expected 0.30 value. The intercept on the $x$ axis is $219.8 \mathrm{~ms}$, a value similar, though somewhat smaller, than the previously reported $300 \mathrm{~ms}$. Therefore, for lexicaldecision tasks, the same linear relationship holds for both control and dyslexic children that is predicted by the DEM. No increase in fit was observed when a second- or third-order polynomial was considered (as tested with the Akaike information criterion [AIC]; Akaike, 1973).

Figure 2 plots $S D$ s as a function of the condition means for the reading-aloud tasks of dyslexic and control readers (for values of the condition means, please refer to Table $2 \mathrm{a}$ in Zoccolotti et al., 2015). Several aspects of this plot are consistent with the DEM. First, the data points for control and dyslexic children lie on the same regression line. This fits with the DEM's prediction that the regression between means and SDs will be independent of group membership. Second, the regression line between the means and $S D$ s accounts for a sizeable amount of variance $\left(R^{2}=.82\right)$, a relevant finding in light of the fact that experimental points come from 11 different studies. Third, if a third-order polynomial is fit to the data, there is a significant increase in the explained variance $\left(R^{2}=.85\right.$; with the AIC value reducing from 1,549 to $1,529, p<.001)$.

The presence of an initial positive acceleration is predicted by the DEM (see, in particular, Eq. 7 in Myerson et al., 2003), although in the present data this was captured by a third-order polynomial, rather than the second-order polynomial posited by the model. Explaining this difference is beyond the scope of the present article. In any case, the fit captures the tendency for $S D$ s to flatten out with the shortest (and longest) means.

Notably, some aspects are at variance with the data on lexical decision presented above. First, the slope of the linear regression (0.66) is about twice as large as those typical of other timed tasks (see the fits reported by Myerson et al., 2003). Second, the intercept of the linear regression on the $x$-axis (482.6 ms) is greater than we would expect on the basis of Myerson et al.'s fits (i.e., $300 \mathrm{~ms}$ ). To appreciate the difference from the data in Fig. 1, the same ratio between the abscissa and the ordinate is kept in the two figures; for a visual comparison between the reading and lexical-decision tasks, see Fig. 3, which reports the two regression lines as well as the intercepts on the $x$-axis.

As a control (not presented in the figure), we examined whether the relationship between $S D$ s and condition means changed when data were added from the pseudoword conditions (Table 2). In general, the patterns of results were similar for both the linear regression $\left(y=0.73 x-372.9, R^{2}=.82\right.$; indicating an intercept on the $x$-axis of $508.0 \mathrm{~ms}$ ) and the polynomial solution $\left(y=-5 \mathrm{E}-07 x^{3}+0.002 x^{2}-1.4 x+\right.$ $338.9, R^{2}=.89$ ), with the AIC value reducing from 2,067 for the linear model to 2,039 for the cubic model $(p<.001)$.

Overall, the children's data for both lexical decision and reading aloud fit well with the DEM predictions, as do those of adults, in keeping with the generality of the model.

The main new finding in the present study is that interindividual variability grows at a much faster rate as a function of condition difficulty for reading aloud than for lexical-decision tasks. In line with previous analyses by Myerson et al. (2003) and the data from Chen et al. (2007), lexical-decision tasks yielded a relationship approximately consistent with the 0.30 
Table 1 Mean decision times (in milliseconds) and SDs for children with dyslexia and typically developing children tested in lexical-decision studies

Reference and Conditions
Children With Dyslexia

Mean $S D$

Typically Developing Children

Mean SD

Di Filippo et al. (2006)

32 dyslexics and 86 controls; $3 r d$ and 4 th grade

Short words

1,852

Long words

Short pseudowords

Long pseudowords

Partial mean

Marinelli, Angelelli, Notarnicola, \& Luzzatti, (2009)

13 dyslexics and 57 controls; 5th grade§

Short high-frequency (HF) irregular words

Long HF irregular words

Short low-frequency (LF) irregular words

Long LF irregular words

Short HF regular words

Long HF regular words

Short LF regular words

Long LF regular words

Short HF irregular fakes

Long HF irregular fakes

Short LF irregular fakes

Long LF irregular fakes

Short HF regular fakes

Long HF regular fakes

Short LF regular fakes

Long LF regular fakes

Partial mean

Marinelli et al. (2011)

23 dyslexics and 42 controls; $4^{\text {th }}$ grade

Pseudowords

LF words

HF words

Partial mean

Paizi et al. (2013)

17 dyslexics and 17 controls; $6^{\text {th }}$ grade

HF words

LF words

Pseudowords (from LF words)

HF 4-letter words

HF 5-letter words

HF 6-letter words

HF 7-letter words

LF 4-letter words

LF 5-letter words

LF 6-letter words

LF 7-letter words

Pseudowords (from HF 4-letter words)
2,876

2,278

1,938

1,184

1,391

1,645

1,743

2,391

3,276

2,482

2,128

2,465

2,322

2,647

2,005

2,483

2,056

2,585

2,659

3,024

3,079

3,401

2,916

3,205

2,998

3,643

2,726

2,364

1,164

1,242

1,348

1,325

1,298

1,349

1,396

1,522

1,558
519

616

594

636

828

900

770

797

658

836

834

1,033

836

1,096

817

1,137

961

803

1,104

943

1,147

927

901

1,742

1,501

1,267

1,504

1,378

1,569

1,862

1,692

1,976

1,355

1,785

1,383

1,800

1,912

2,224

2,123

2,088

1,902

2,062

2,007

2,155

1,868
266

307

338

479

544

791

623

662

450

671

396

679

788

884

888

889

610

689

809

748

562

426

303

92

333

716

815

879

874

715

711

758

734

803

803

805

806

868
100

164

151

115

108

105

115

118

121

114

125

121 
Table 1 (continued)

Reference and Conditions

Children With Dyslexia

\begin{tabular}{ll}
\hline Mean \\
1,716 \\
1,967 \\
2,085 & 438 \\
1,619 & 520 \\
1,775 & 615 \\
1,918 & 411 \\
2,069 & 474 \\
1,566 & 580
\end{tabular}

1,84

1,824

1,883

2,789

3,319

3,536

2,279

2,583

2,805

1,801

2,068

2,323

1,321

1,413

1,359

2,086

2,596

2,697

1,859

2,031

2,410

1,618

1,624

1,855

2,164

2,155

629
Typically Developing Children

\begin{tabular}{ll}
\hline Mean & $S D$ \\
\hline 927 & 138 \\
905 & 147 \\
988 & 210 \\
885 & 142 \\
901 & 151 \\
921 & 156 \\
979 & 173 \\
840 &
\end{tabular}

6-letter high-frequency words (BC)

Total mean ${ }^{\circ}$

$S D$

$\begin{array}{ll}438 & 927 \\ 520 & 905 \\ 615 & 988 \\ 411 & 88 \\ 474 & 90 \\ 580 & 92 \\ 551 & 979 \\ & 840\end{array}$

$1,195 \quad 353$

$1,272 \quad 462$

$1,258 \quad 515$

$1,922 \quad 705$

$1,997 \quad 732$

$2,379 \quad 814$

$1,368 \quad 411$

$1,526 \quad 611$

$1,563 \quad 669$

$1,281 \quad 461$

$1,293 \quad 393$

$1,406 \quad 500$

$984 \quad 386$

$979 \quad 372$

$1,058 \quad 389$

$1,402 \quad 581$

$1,498 \quad 557$

$1,681 \quad 668$

$1,218 \quad 367$

$1,215 \quad 430$

$1,336 \quad 567$

$1,087 \quad 381$

$1,103 \quad 406$

$1,156 \quad 425$

1,382

1,345

447

Numbers of participants and the experimental conditions are listed for each study. ${ }^{\circ}$ Total means do not include partial means. § Additional data were collected after the completion of Marinelli et al.'s (2009) study using the same procedure and sample inclusion criteria. Data from this enlarged sample are presented here. * Although only data from the mixed- block conditions were published, both types of manipulations were run at the time of the experiment and are presented here.

slope. By contrast, reading tasks generated a much steeper slope and a considerably greater intercept on the $x$-axis. The data for the two sets of tasks were drawn from studies from a relatively homogeneous subject population and cut across a wide range of stimulus manipulations. Thus, it seems unlikely that such a large difference would be accidental. Therefore, it would be interesting to understand why the relationship, which is considered to be the underlying rule governing individual differences in timed tasks, changes so much when individuals make lexical decisions versus when they read aloud. 
Table 2 Pseudoword reading aloud in dyslexic and skilled children: Mean reading times (in milliseconds) and SDs of pseudowords for children with dyslexia and typically developing children tested in reading studies (derived from the list provided in Zoccolotti et al., 2015)

\begin{tabular}{|c|c|c|c|c|}
\hline \multirow[t]{2}{*}{ Reference and Conditions } & \multicolumn{2}{|c|}{ Children With Dyslexia } & \multicolumn{2}{|c|}{ Typically Developing Children } \\
\hline & Mean & $S D$ & Mean & $S D$ \\
\hline \multicolumn{5}{|c|}{ Burani, Marcolini, De Luca, \& Zoccolotti (2008) } \\
\hline \multicolumn{5}{|c|}{17 dyslexics and 34 controls; 6th grade } \\
\hline Complex pseudowords (Exp1) & 1,400 & 875 & 770 & 191 \\
\hline Simple pseudowords & 1,739 & 1,185 & 836 & 209 \\
\hline \multicolumn{5}{|l|}{ Zoccolotti et al. (2008) } \\
\hline \multicolumn{5}{|c|}{26 dyslexics and 81 controls; 6-7-8th grade } \\
\hline 4-letter pseudoword & 1,172 & 489 & 676 & 113 \\
\hline 5-letter pseudoword & 1,510 & 745 & 764 & 198 \\
\hline 6-letter pseudoword & 1,558 & 765 & 753 & 196 \\
\hline 7-letter pseudoword & 1,908 & 1,093 & 845 & 234 \\
\hline 8-letter pseudoword & 1,899 & 921 & 869 & 268 \\
\hline \multicolumn{5}{|l|}{ Paizi et al. (2011) } \\
\hline \multicolumn{5}{|c|}{18 dyslexics and 36 controls; 6th grade } \\
\hline 4-letter pseudoword, pure list & 806 & 172 & 614 & 67 \\
\hline 5-letter pseudoword, pure list & 859 & 192 & 635 & 87 \\
\hline 6-letter pseudoword, pure list & 940 & 222 & 678 & 101 \\
\hline 7-letter pseudoword, pure list & 981 & 219 & 699 & 121 \\
\hline 4-letter pseudoword, mixed list & 825 & 194 & 611 & 69 \\
\hline 5-letter pseudoword, mixed list & 882 & 220 & 635 & 81 \\
\hline 6-letter pseudoword, mixed list & 997 & 286 & 688 & 108 \\
\hline 7-letter pseudoword, mixed list & 1,039 & 287 & 705 & 117 \\
\hline \multicolumn{5}{|l|}{ Paizi et al. (2013) } \\
\hline \multicolumn{5}{|c|}{17 dyslexics and 34 controls; 6th grade } \\
\hline HF derived pseudowords & 899 & 167 & 637 & 51 \\
\hline LF derived pseudowords & 965 & 154 & 657 & 57 \\
\hline \multicolumn{5}{|l|}{ Martelli et al. (2014) } \\
\hline \multicolumn{5}{|c|}{25 dyslexics and 43 controls; 6th grade } \\
\hline 4-letter pseudoword & 943 & 493 & 528 & 85 \\
\hline 5-letter pseudoword & 1,121 & 591 & 569 & 123 \\
\hline 6-letter pseudoword & 1,232 & 697 & 611 & 135 \\
\hline 7-letter pseudoword & 1,367 & 808 & 639 & 133 \\
\hline
\end{tabular}

Numbers of participants and the experimental conditions are listed for each study

Clearly, the source of the effect cannot be in the nature of the stimuli, which are orthographic for both the reading and the lexical-decision tasks. Note that inclusion of the pseudoword conditions did not appreciably change the pattern of results for reading. Therefore, the presence of pseudowords in lexical-decision tasks is not what accounts for the difference between the two tasks.

More generally, this is not keeping with the observation that the same 0.30 slope applies to a large variety of linguistic and visuospatial tasks (Chen et al., 2007; Myerson et al., 2003). Alternatively, it might be the type of response that makes the difference. Although lexical-decision tasks (and all other tasks considered by Chen et al., 2007, and Myerson et al., 2003) involve a manual motor response, reading requires a vocal response. We believe that this interpretation is unlikely. We recently completed a study in which we compared children with either dyslexia, dyscalculia, or both to a group of typically developing children in a set of reading and calculation tasks that required exclusively vocal responses (Di Filippo \& Zoccolotti, 2017). In keeping with the present findings, the slopes of the relationship were 0.76 for the reading tasks and 0.27 for the calculation tasks (which included tasks such as making small sums or subtractions or judging which of two numbers was larger). Thus, the dissociation between the slopes 


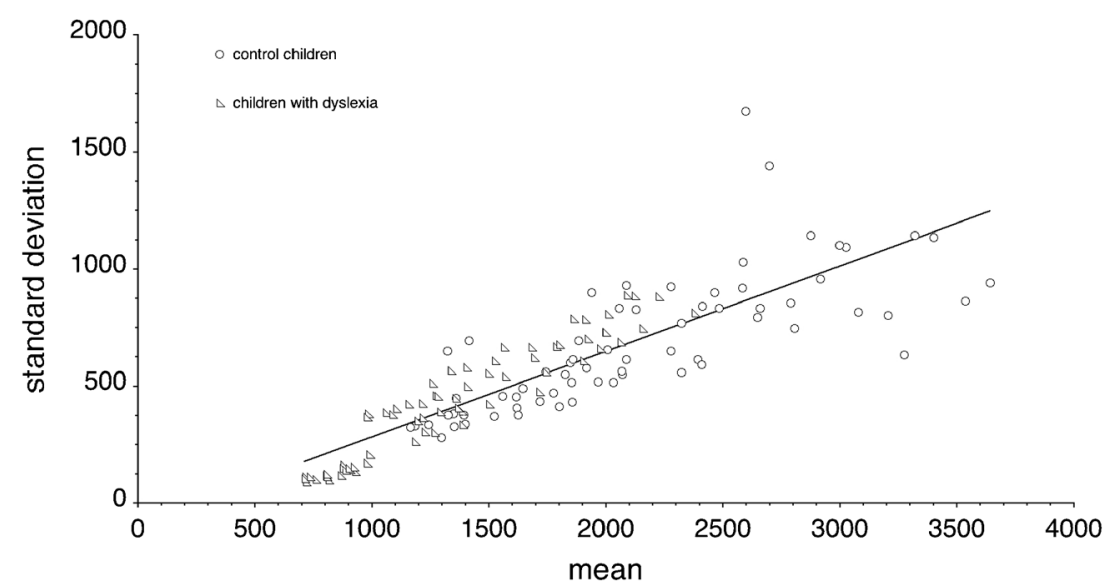

Fig. 1 Lexical-decision tasks. Test of the difference engine model (DEM) predictions, based on the results of dyslexic (open triangles) and control (open circles) children's groups in several lexical-decision conditions (see Table 1). Standard deviations across individuals are

for reading and other timed cognitive tasks is present also when the motor output of the response is held constant.

Nevertheless, the type of response output may be responsible for the longer $x$-intercept found in the present study for reading than for lexical-decision tasks; in fact, the intercept represents an estimation of the time of the response that is due to the sensory-motor compartment (Myerson et al. 2003), and whereas the sensory components were comparable, the motor responses in the two tasks were different (voice vs. hand). Finally, it seems unlikely that the age difference between the groups of children tested with the lexical-decision (third to fifth grade) and reading-aloud (sixth grade) tasks produced the difference. Indeed, the data of children in lexicaldecision tasks showed very similar parameters to those of both younger and older adults, in keeping with the generality of the relationship between means and $S D$ s predicted by the DEM. plotted as a function of the overall group means for the same conditions. The linear regression fit $\left(y=0.36 x-80.2, R^{2}=.73\right)$ is shown. The intercept on the $x$-axis is $219.8 \mathrm{~ms}$

As for reading, we only presented data on sixth grade children because a large number of children/conditions were available for this age (and were already listed as part of a previous reanalysis). However, we were able to trace one study involving third-grade children (Zoccolotti, De Luca, Gasperini, Judica, \& Spinelli, 2005) and two involving fourth-grade children (Marinelli et al., 2011; Marinelli, Traficante, Zoccolotti, \& Burani, 2013), for a total of seven conditions and 168 subjects (114 controls and 54 children with dyslexia). The fit for this small set of data was quite similar to that of the larger set of sixth-grade children ( $\left.y=0.68 x-370.4, R^{2}=.76\right)$, yielding an $x$-intercept of $543.3 \mathrm{~ms}$. These data are in keeping with the idea that the difference between lexical-decision and reading tasks cannot be easily explained in terms of age differences.

More interesting alternative interpretations arise if we consider the general nature of reading and lexical-decision (and

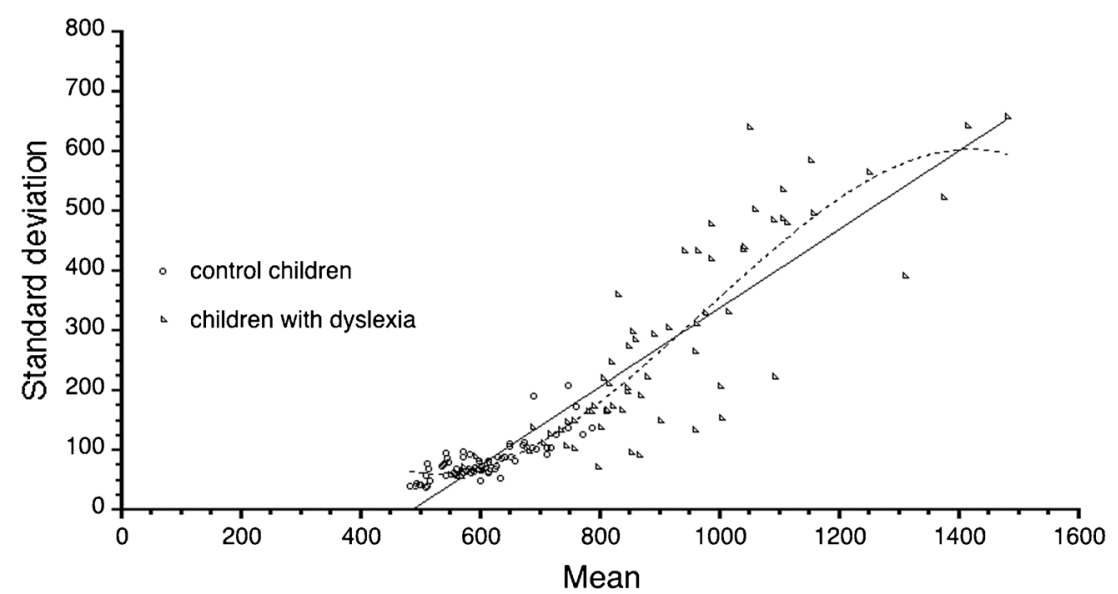

Fig. 2 Reading-aloud tasks. Test of the DEM predictions, based on the results of sixth-grade dyslexic (open triangles) and control (open circles) children of the same age in several word-reading-aloud conditions (source data are from Table 2a in Zoccolotti et al., 2015). Standard deviations across individuals (dyslexics and controls) are plotted as a function of the overall group means for the same conditions. The linear regression fit $(y=0.66 x-317.4)$ has a determination coefficient of 0.82 and an intercept on the $x$-axis of $482.6 \mathrm{~ms}$. The fit of a third-order polynomial is also shown $\left(y=-2 \mathrm{E}-06 x^{3}+0.0045 x^{2}-\right.$ $\left.3.4 x+855.1, R^{2}=.85\right)$ 


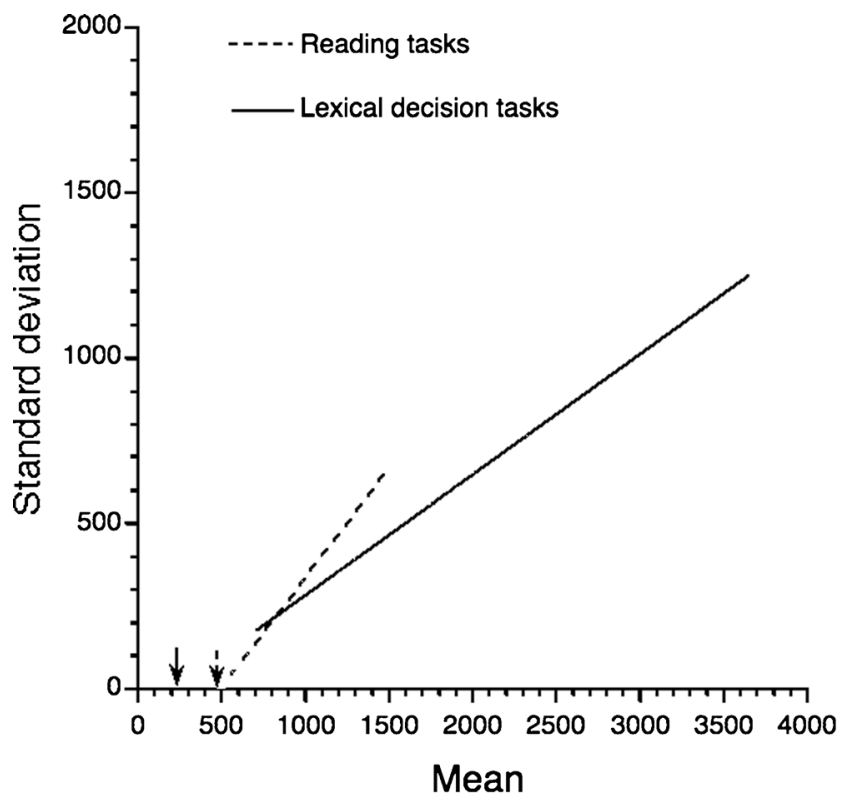

Fig. 3 Linear regressions for reading and lexical-decision tasks are displayed together to highlight the different slopes and intercepts on the $x$-axis of the two sets of data

other timed) tasks. Similar (though not identical) psycholinguistic factors affect performance on reading and lexicaldecision tasks; however, the latter places a heavier weight on the decisional component (Yap \& Balota, 2015). Thus, at some point in the course of processing, the observer has to decide between two alternatives. Consistent with this, models that try to account for performance on lexical-decision tasks (and for performance on other tasks featuring a fixed number of alternatives) consider as parameters not only sensitivity in performance, but also response criterion (e.g., the diffusion model; Ratcliff, Gomez, \& McKoon, 2004). At the same time, it is probably not accidental that the diffusion model has not yet been applied to reading tasks; in fact, due to the very large number of alternative responses, it seems difficult to frame the response criterion concept for reading tasks.

In reading, the observer has to decide among many (in fact, thousands of) alternatives. Thus, reading requires a close correspondence (or transcoding) between the output of an orthographic analysis and the identification of the corresponding phonological code. By contrast, lexical decision requires similar orthographic processing but does not require coupling the orthographic analysis with a specific phonological output. It does, however, require additional cognitive processing to produce the yes/no decision, and the duration of the latter processing may have a low correlation with the duration of orthographic processing. According to the DEM, the slope of the regression between means and $S D$ s indicates the degree of correlation among the durations of the processing stages. As a working hypothesis, we propose that the requirement for a close correlation between orthographic and phonological processing is what drives the particularly steep relationship between performance and interindividual variability observed in reading tasks in children (slope: 0.66).

In this view, defective reading (and, probably, early reading acquisition) is characterized by a particularly high correlation among the durations of the processing stages in the reading task. Notably, this perspective runs counter to the widespread view that many dyslexic children may be impaired in selective stages of processing (e.g., in phonological processing). By contrast, it has some similarity with the idea that orthographic-phonological binding may hold the key to understanding successful and deranged reading (Blomert, 2011). More generally, these observations seem to be consistent with the perspective that cognitive performance progresses through selforganization (Van Orden, Holden, \& Turvey, 2003; for an application of this approach to dyslexia, see Holden, Greijn, van Rooij, Wijnants, \& Bosman, 2014).

Regardless of the interpretation, the present findings indicate for the first time that defective reading is characterized by a sharp increase in interindividual variability as a function of condition difficulty, more than in other timed tasks (including lexical decision). Ever since the first systematic report of developmental dyslexia (Pringle Morgan, 1896), it has been a frequent clinical observation that even minimal changes in stimulus difficulty may generate a dramatic worsening in a child's performance. Thus, understanding the peculiarity of this characteristic of the reading task may hold the key for a deeper understanding of this learning difficulty.

Author note We thank Marialuisa Martelli for helpful comments on this work, and Claire Montagna for assistance with the English editing of the manuscript.

\section{References}

* Studies with lexical-decision data included in the cross-experiment analysis.

Akaike, H. (1973). Information theory and an extension of the maximum likelihood principle. In B. N. Petrov \& F. Csáki (Eds.), Second international symposium on information theory (pp. 267-281). Budapest: Akademai Kiado.

Blomert, L. (2011). The neural signature of orthographic-phonological binding in successful and failing reading development. NeuroImage, 57, 695-703.

Burani, C., Marcolini, S., De Luca, M., \& Zoccolotti, P. (2008). Morpheme-based reading aloud: Evidence from dyslexic and skilled Italian readers. Cognition, 108, 243-262. doi:10.1016/j.cognition. 2007.12.010

Cerella, J., Poon, L. W., \& Williams, D. M. (1980). Age and the complexity hypothesis. In L. W. Poon (Ed.), Aging in the 1980s (pp. 332-340). Washington, DC: American Psychological Association.

Chen, J., Hale, S., \& Myerson, J. (2007). Predicting the size of individual and group differences on speeded cognitive tasks. Psychonomic Bulletin \& Review, 14, 534-541. 
De Luca, M., Burani, C., Paizi, D., Spinelli, D., \& Zoccolotti, P. (2010). Letter and letter-string processing in developmental dyslexia. Cortex, 46, 1272-1283.

* Di Filippo, G., De Luca, M., Judica, A. Spinelli, D., \& Zoccolotti, P. (2006). Lexicality and stimulus length effects in Italian dyslexics: role of over-additivity effect. Child Neuropsychology, 12, 141-149.

Di Filippo, G., \& Zoccolotti, P. (2017). Isolating global components in developmental dyscalculia and dyslexia. Manuscript in preparation.

Faust, M. E., Balota, D. A., Spieler, H. D., \& Ferraro, F. R. (1999). Individual differences in information-processing rate amount: Implications for group differences in response latency. Psychological Bulletin, 125, 777-799.

Hale, S., \& Myerson, J. (1996). Experimental evidence for differential slowing in the lexical and nonlexical domains. Aging, Neuropsychology, and Cognition, 3, 154-165.

Holden, J. G., Greijn, L. T., van Rooij, M. M. J. W., Wijnants, M. L., \& Bosman, A. M. T. (2014). Dyslexic and skilled reading dynamics are self-similar. Annals of Dyslexia, 64, 202-221.

Lawrence, B., Myerson, J., \& Hale, S. (1998). Differential decline of verbal and visuospatial processing speed across the adult life span. Aging, Neuropsychology, and Cognition, 5, 129-146.

* Marinelli, C. V., Angelelli, P., Notarnicola, A., \& Luzzatti, C. (2009). Do Italian dyslexic children use the lexical reading route efficiently? An orthographic judgment task. Reading and Writing, 22, 333-351.

* Marinelli, C. V., Angelelli, P., Di Filippo, G., \& Zoccolotti, P. (2011). Is developmental dyslexia modality specific? A visual-acoustic comparison on Italian dyslexics. Neuropsychologia, 49, 1718-1729.

Marinelli, C. V., Traficante, D., Zoccolotti, P., \& Burani, C. (2013). Orthographic neighborhood-size effects on the reading aloud of Italian children with and without dyslexia. Scientific Studies of Reading, 17, 333-349.

* Marinelli, C. V., Traficante, D., \& Zoccolotti, P. (2014). Does pronounceability modulate the letter string deficit of children with dyslexia? Evidence from lexical decision task. Frontiers in Psychology, 5, 1353. doi:10.3389/fpsyg.2014.01353

Martelli, M., De Luca, M., Lami, L., Pizzoli, C., Pontillo, M., Spinelli, D., \& Zoccolotti, P. (2014). Bridging the gap between different measures of the reading speed deficit in developmental dyslexia. Experimental Brain Research, 232, 237-252. doi:10.1007/s00221013-3735-6

Myerson, J., Hale, S., Zheng, Y., Jenkins, L., \& Widaman, K. F. (2003). The difference engine: A model of diversity in speeded cognition. Psychonomic Bulletin \& Review, 10, 262-288.
Paizi, D., Burani, C., De Luca, M., \& Zoccolotti, P. (2011). List context manipulation reveals orthographic deficits in Italian readers with developmental dyslexia. Child Neuropsychology, 17, 459-482. doi:10.1080/09297049.2010.551187

* Paizi, D., De Luca, M., Zoccolotti, P., \& Burani, C. (2013). A comprehensive evaluation of lexical reading in Italian developmental dyslexics. Journal of Research in Reading, 36, 303-329.

Pringle Morgan, W. (1896). A case of congenital word-blindness. British Medical Journal, n. 1871, 1378.

Puopolo, C., Martelli, M., \& Zoccolotti, P. (2013). Role of sensory modality and motor planning in the slowing of patients with traumatic brain injury: A meta-analysis. Neuroscience \& Biobehavioral Reviews, 37, 2638-2648.

Ratcliff, R., Gomez, P., \& McKoon, G. (2004). A diffusion model account of the lexical decision task. Psychological Review, 111, 159182. doi:10.1037/0033-295X.111.1.159

Van Orden, G. C., Holden, J. G., \& Turvey, M. T. (2003). Selforganization of cognitive performance. Journal of Experimental Psychology: General, 132, 331-350.

Verhaeghen, P. (2013). The elements of cognitive aging: Meta-analyses of age-related differences in processing speed and their consequences. New York: Oxford University Press.

Wagenmakers, E.-J., \& Brown, S. (2007). On the linear relation between the mean and the standard deviation of a response time distribution. Psychological Review, 114, 830-841. doi:10.1037/0033-295X.114. 3.830

Yap, M. J., \& Balota, D. A. (2015). Visual word recognition. In A. Pollatsek \& R. Treiman (Eds.), The Oxford handbook of reading (pp. 26-43). New York: Oxford University Press.

Zoccolotti, P., De Luca, M., Gasperini, F., Judica, A., \& Spinelli, D. (2005). Word length effect in early reading and in developmental dyslexia. Brain and Language, 93, 369-373.

Zoccolotti, P., De Luca, M., Judica, A., \& Spinelli, D. (2008). Isolating global and specific factors in developmental dyslexia: A study based on the rate and amount model (RAM). Experimental Brain Research, 186, 551-560.

Zoccolotti, P., De Luca, M., \& Spinelli, D. (2015). Discrete versus multiple word displays: A re-analysis of studies comparing dyslexic and typically developing children. Frontiers in Psychology, 6, 1530. doi: 10.3389/fpsyg.2015.01530 